\title{
Primera protesta estudiantil médica en Perú
}

\author{
Eduardo Zarate ${ }^{1}$ \\ ${ }^{\text {I} F a c u l t a d ~ d e ~ M e d i c i n a ~ U N M S M . ~ D e p a r t a m e n t o ~ d e ~ M e d i c i n a ~ P r e v e n t i v a ~ y ~ S a l u d ~ P u ́ b l i c a . ~}$
}

\begin{abstract}
Resumen
Los años iniciales de la República, 1821-1830, estuvieron signados por continuas guerras civiles y conflictos internacionales, con una agricultura y comercio exterior paralizados, lo que originó grave crisis fiscal. En este marco societal, el Colegio de Medicina sufrió una grave crisis con periodos de cierres y cambios en su programa de enseñanza inicial. En este contexto se presenta un manuscrito inédito sobre la primera protesta estudiantil de medicina en 1829. Los reclamos eran de corte académico y por mejores condiciones de estudio, los cuales no fueron atendidos por las autoridades del colegio.
\end{abstract}

Palabras clave: Estudiantes de medicina; historia de la medicina, Perú; escuelas médicas.

\section{First medical students’ protest in Peru Summary}

The early years of the Peruvian Republic (1821 - 1830) were characterized by continuous civil wars and international conflicts that stalled agriculture and commerce and originated a serious fiscal crisis. With this social environment frame, the College of Medicine suffered a serious crisis with shut-down periods and changes in academic programs. Within this context, an unpublished manuscript on the first medical students' civil protest was presented in 1829 . The claims refered to academic issues and better learning conditions, claims unattended by the College authorities.

Key words: Students, medical; history of medicine, Peru; schools, medical.

\section{Introducción}

Una mirada a la actual respuesta educativa de las escuelas de medicina frente a los problemas sanitarios

del país nos muestra una respuesta divergente, un sector educativo fragmentado, dislocado. Luego de décadas de un crecimiento exponencial, no ha podido producir una respuesta sustancial al modelo de atención de la salud del país. Las causas del fracaso del sistema educativo estriba en la ausencia de una comprensión adecuada de la dinámica de los cambios societales y en la formulación constructiva de los sistemas sanitarios. ¿Cómo se configura la escuela de medicina en sus inicios? ¿La creación del colegio de medicina fue una respuesta a un problema sanitario?. Estimo que una parte de

Correspondencia:

Dr. Eduardo Zárate Cárdenas

Facultad de Medicina. UNMSM

Av. Grau 755. Lima 1 - Perú

E-mail:eduzac@terra.com.pe las respuestas podrá ser obtenida al estudiar el proceso de formación del Colegio Real de Medicina y Cirugía realizado por Hipólito Unanue y su grupo en los años finales de la colonia ${ }^{1}$.

En este contexto, se realiza un estudio siguiendo el postulado que la rotura de la respuesta educativa en el Perú es resultado de un proceso histórico cuyos elementos centrales fueron propuestos a inicios del siglo XIX. El proceso formativo de la escuela de medicina de Lima fue deformado y reformulado en medio de guerras civiles entre bandos de militares. Será bajo el manto

\footnotetext{
1 La célula real que creo el Colegio Real de Medicina y Cirugía fue dada el 15 de mayo por el Rey Fernando VII.
} 
de una severa crisis política y económica de los años iniciales de la república en el cual se desarrolló el Colegio de Medicina de Lima. En la medida que las luchas intestinas de caudillos militares amainaran el clima político e intelectual, el Colegio de Medicina podrá contar con recursos económicos mínimos que le permitirán avanzar hasta lograr constituirse en el primero en Sudamérica bajo la égida del profesor Cayetano Heredia.

Por lo tanto, esta investigación busca estudiar la transformación inicial de la educación médica en el Perú, bajo la tesis de que dicha transformación fue parte constitutiva de un proceso mayor, la primera modernización del estado peruano, y que la configuración resultante concretó cambios en el posicionamiento de los médicos como parte importante de la sociedad peruana en medio de los cambios económicos, derivados de un reacomodo del Perú en el contexto económico internacional.

Las primeras décadas de la república fueron de continuas guerras de civiles y facciones de militares ${ }^{2}$, complots y divisiones entre republicanos y monárquicos. En medio de estos años se va a ir conformando la institución educativa médica peruana. Esta situación se verá agravada con problemas de salud pública, como son las epidemias de viruela y cólera.

En la primera década republicana, en el plano educativo se producirán cierres parciales del Colegio de la Independencia (ex -Colegio Real de Medicina y Cirugía.) y la primera protesta pública de los estudiantes contra la administración del rector José M Dávila. Una pesquisa de los años iniciales del Colegio de la Independencia (CI) no puede dejar de lado a los actores casi siempre olvidados, los estudiantes y sus propuestas.

La casi ausencia de trabajos para el periodo de 1821-30 por los estudiosos de la historia de la medicina, se podría explicar porque en todos los autores despierta más interés tratar solo épocas de bonanza y esplendor como las del gobierno de Cayetano Heredia. Paz Soldán CE evita presentar estos años y sólo anota que después de años de tanteos y ensayos la conducción del Colegio de la Independencia fue confiada a Cayetano Heredia ${ }^{3}$. Lastres $\mathbf{J}$, al tratar sobre la enseñanza médica en estos años, reduce el tratamiento a pocos párrafos, ocupándose más sobre los personajes que ocuparon los cargos directivos ${ }^{4}$. H Valdizán divide este periodo en dos etapas: de 1821 a 1825 y de 1826 a 1830. Para el primer periodo indica que en los archivos del CI sólo marcan celebración de ceremonias de índole políticas.

Nuestra investigación no ha encontrado documentos sobre estos años que muestren una continuidad académica y, al igual que Valdizán, sostenemos que hubo años de clausura y años durante los cuales el CI hizo una vida precaria ${ }^{5}$. Esta precaria situación académica es determinada por 1.- su líder H Unanue y sus principales seguidores Gregorio Paredes y Miguel Tafur pasaron a ocupar cargos públicos; el elemento unificador de la promesa de un colegio que creara una Medicina Peruana se diluyó porque el grupo inicial asume la retórica política y electoral; y, 2.la crisis económica nacional. Esto va a quedar evidenciado porque al final de la década de 1820 no hubo división de grupos, sino que el nuevo grupo de dirigentes -entre ellos Nicolás de Piérola y José M Dávila- no estuvieron comprometidos con la propuesta inicial y sólo fueron atentos a aceptar los requerimientos del gobierno de turno.

2 Aljobin Cristóbal. Ha sostenido que en el Perú durante el periodo 1821-1845 existieron siete constituciones, diez congresos, tres guerras internacionales cincuenta y tres gobiernos e innumerables rebeliones y revoluciones. Caudillos y Constituciones en el Perú 1821-1845. Lima Perú: Fondo de Cultura Económica PUCP 2000. pp.40.

Paz Soldan CE. La escuela medica peruana. pp. 32-33. Lima. Perú: Editorial Imprenta del Hospital Víctor Larco Herrera.

4 Lastres J. Historia de la medicina peruana. La medicina en la Republica. Pp. 77. Lima Perú: Universidad Nacional Mayor de San Marcos Imprenta Santa Maria, 1951.

5 Valdizán H. La facultad de medicina de Lima 1811-1911, pp. 147. Lima: Imprenta Sanmarti y Cia. 1913. 
En los primeros años de la república, José de San Martín impulsó las ideas liberales, como la educación primaria gratuita y obligatoria, el otorgamiento de becas para los alumnos pobres y la implantación en todas las escuelas del método de enseñanza Lancasteriano. Las escuelas primarias que existían en la Colonia eran pocas y con maestros escasamente preparados, por lo que estaban abandonadas.

\section{Las crisis económicas y el Colegio de la Independencia}

Los primeros años del colegio de medicina fueron con problemas de orden económico, académicos y administrativos. Las primeras dificultades económicas fueron que el ejecutivo no tenia fondos para asistir a los enfermos de los hospitales y menos para los sueldos de los profesores de los colegios. Expresión de esta situación es el decreto del 31 de octubre de 1822 dado por el congreso constituyente, en el cual la junta gubernativa ordena se ponga en actividad sin demora el colegio San Carlos, que se halla en total decadencia, y ordena a la administración entregar mensualmente la cantidad de 985 pesos y dos reales y medio para sus gastos. Para el año siguiente (enero de 1823), la crisis abarcó a los alumnos del colegio de medicina y se expresó en la dificultad de éstos para realizar los pagos para los gastos de su graduación, lo que obligó al congreso constituyente dictar el decreto para exonerar del pago de derechos de graduación a alumnos pobres. Para graficar la continuidad de la crisis económica, al final del año 1826, el rector Nicolás de Piérola se quejaba de la escasez de material bibliográfico y de laboratorio “...una gran porción de los primeros están apolillados ....los segundos, casi todos en absoluta inutilidad con las piezas sueltas y muchas perdidas..." Sólo era rescatable en buen estado una brújula descompuesta, un teodolito y un anteojo grande de reflexión ${ }^{6}$. A este estado de abandono, inoperancia académica y mundo de banalidades se agregaron las dudas sobre el manejo económico de la administración del ex rector D. José Mariano Fernandini. El rector ofició, en diciembre de 1826, al ministro a fin de que se obligue al rector cesante presentar las cuentas de los años que ha administrado en el colegio, haciendo saber que lo solicitado ha obtenido respuestas dilatorias. El proveído del ministro indicó “...exíjanle las rentas al Dr. Fernandini..." 7 .

El Colegio de la Independencia, como institución del aparato gobernativo, estaba sujeto a las disposiciones del prefecto departamental, quien indicó al rector que debería asistir, junto con los miembros de su corporación, a la función religiosa que se haría en la Catedral ${ }^{8}$. Estas disposiciones de asistencia a misa, ceremonias en palacio de gobierno, juramentaciones de nuevos gobiernos, eran solicitadas por el prefecto departamental con carácter obligatorio, las cuales se repitieron a lo largo de la década. Como anota J Basadre, el Perú se empobreció notablemente durante la guerra de la independencia; las diversas luchas le arrancó dinero, hombres, materiales. El desembarco de la expedición de San Martín dio lugar que el comercio exterior quedara anulado y en el interior los reclutamientos de hombres, los empréstitos forzados o no y la inseguridad arruinó la agricultura, la minería y las industrias. La guerra duró cuatro largos años ${ }^{9}$.

El ejército del virrey La Serna, al retirarse, causaba destrozos e incorporaba, por medio de la fuerza, a los indios en sus ejércitos, con lo cual agravaba la precaria situación económica. $\mathrm{H}$ Unanue, en su memoria de Ministro de Hacienda en 1825, manifiesta que en el país ha desaparecido la agricultura, la minería está derrumbada y el comercio de la capital sin puertos. El siguiente ministro dio un diagnóstico similar, indicando que

\footnotetext{
AGN Colegio de la Independencia. RJ L 178, 30 diciembre 1826.

AGN Colegio de la Independencia. RJ L 10, 4 diciembre 1826.

DFML 25 diciembre 1826.

Basadre J. Historia de la Republica del Peru tomo I p.136.
} 
los hombres arrancados de sus hogares e incorporados a los ejércitos hacían falta en los campos. La inseguridad, la anarquía y el desorden eran los elementos que impedían el desarrollo de cualquier actividad productiva. En medio de esta situación general del país era lógico que una naciente institución, sin liderazgo interno y sin voz en la escena pública tuviera un papel venido a menos y careciera de fondos económicos. Los tiempos eran de guerras, epidemias y economía quebrada, por lo que el colegio de medicina fue sólo un acompañante a ceremonias para rendir tributos a los gobiernos de turno. Durante febrero 1827, el rector del Colegio de la Independencia dirigió oficios al Ministro indicando que en estos meses sólo tienen como ingreso adicional lo que pagan cuatro internos pensionistas, a 150 pesos cada uno. Sus gastos en total son 13,044 pesos teniendo un déficit de 3,020 pesos ${ }^{10}$. Las rentas del gobierno eran nominales, sin una garantía de poderlas efectivizar y, en el caso de entregar pequeñas sumas, éstas eran para mantener lo mínimo. Como medida para no cerrar el colegio, se procedió a dar horas libres a los alumnos para que tomaran sus alimentos en sus casas; el régimen de internado fue haciéndose flexible por razones económicas. No fueron suficientes los reclamos del rector ni los del Protomédico del Estado. En noviembre 1828, el rector José M Dávila ${ }^{11}$ se dirige al ministro indicando que no ha recibido la asignación correspondiente desde hace seis meses y expresa su extrañeza por el retraso. Como medida para aligerar gastos administrativos, se propone que el dictado de clases esté a cargo de alumnos del último año; por lo cual se encarga la cátedra de física experimental al bachiller Miguel De los Ríos. Este oficio lo firmará el Dr. Miguel Tafur como Protomédico General del Estado y profesor del CI.

Para el año siguiente, 1828, la realidad era que las rentas no llegaban, a pesar de los reiterados oficios del rector José M Dávila. Éste graficará la situación a través del oficio dirigido al ministro: “...la casi obstrucción de las rentas ...teniendo que salir diariamente los alumnos a sus casas para comer; además de perder tiempo, no pueden sujetarse a una disciplina....." ${ }^{12}$. Desde los tiempos del virrey F Abascal, se asignó como rentas del Colegio de la Independencia el producto de una corrida de toros a realizarse en el mes de febrero de cada año, en la plaza de Acho. Al cierre del año 1828, el rector José M Dávila se lamentaba de no haberla podida realizar, dejando de captar esos fondos; lo cual hacía más crítica la situación económica ${ }^{13}$. Los años siguientes fueron de una prolongada crisis económica; los manuscritos revisados insisten en una continuidad por reclamar rentas. El cual siendo el único colegio de Medicina de Sudamérica no consiguió la patrocinio del gobierno, dando origen que permisos de alumnos a sus casas para la toma de alimentos y los cierres temporales del colegio de medicina serán lo cotidiano ${ }^{14}$.

\section{Las epidemias}

En febrero 1822, el gobierno mostró preocupación por la difusión de la vacuna de la viruela, para lo cual ordenó que los curas, antes de acudir a sus curatos ${ }^{15}$, recibirían del Protomédico el fluido vacunal, en número necesario para su parroquia, informarían mensualmente al prefecto del número de niños vacunados y convocarían un día a la semana a las madres para que lleven a sus niños ${ }^{16}$. Al cabo de tres años, esta medida mostró escasos resultados, por lo que, en 1825, mediante circular, el gobierno dispone que, teniendo conocimiento del abandono en que se encuentra la Junta conservadora de la vacuna, delega a la Municipalidad la conservación y la vacunación de la población ${ }^{17}$. En los territorios recuperados a las tropas realistas, la viruela

1 AGN Colegio de la Independencia. Legajo 11 ff. 2,8 de enero de 1827.

AGN Colegio de la Independencia. L 24 cuaderno 119 ff.1 , 13 de noviembre 1828.

AGN Colegio de la Independencia. L 30 cuaderno 94 ff. 320 y 23 de junio de 1829.

AGN Colegio de la Independencia. L 25 numero 54 ff. 3 , 6 de diciembre de 1828.

AGN L. 30 número 9 ff 2 del 23 julio 1829 y L 34 número 245 ff 2 del 24 de abril de 1830. 
asolaba, por lo que se dispuso que todos los prefectos coordinen con el de Lima para generalizar la vacuna en estos territorios e invocó para que se establecieran cementerios en todos los pueblos ${ }^{18}$. Estas medidas de encargar la vacunación a la iglesia y luego a los municipios, hablan del imaginario de gobernantes acerca: de explicaciones de la enfermedad por medios sobrenaturales, la Interrelación entre religión y enfermedad y los vínculos entre la Iglesia y el Estado. En un país desorganizado, con escasas vías de comunicación y analfabeto, el púlpito era el vinculo para trasmitir las ideas del gobierno. En otro plano, el control político de las instituciones a través de los prefectos en cada departamento, los cuales eran una suerte de presidentes departamentales encargados de la ciudad, de las milicias locales y del control de epidemias, contribuyeron a la escasa importancia en la sociedad de las profesiones médicas y del colegio de medicina.

H Unanue, como ministro de gobierno, el 9 de noviembre 1825 dispuso medidas de refuerzo a la vacunación antivariólica, como que los médicos vacunen en los cuarteles y no se admita en los colegios o escuelas a los que no acrediten haberse vacunado. Se ordenaba una reevacuación general con la finalidad de cubrir posibles fallas en la aplicación de la vacuna o de la conservación de ésta o de técnicas aplicadas en la selección del grano. $\mathrm{Al}$ cabo de casi un año, vuelve el gobierno esta vez con decreto del 24 octubre 1826, dado por Andrés de Santa Cruz y José María de Pando, a tratar la pandemia de la viruela esta vez con medidas de policía médica, como exclusión de los virulentos de los hospitales y captura de los enfermos que permanecieran en sus domicilios por comisarios de barrios, llevándolos al hospital de los incurables ${ }^{19}$. Similares medidas fueron tomadas durante la epidemia de peste en Londres en $1665^{20}$.

La viruela fue una constante preocupación de los diversos gobiernos, cada uno ensayando nuevas medidas, más para su imagen o como presión de la situación, sin una coherencia con las anteriores o previas evaluaciones. En 1832, el gobierno de Agustín Gamarra emite una circular a los prefectos de todos los departamentos "...los estragos de la peste de la viruela, que está causando en las poblaciones deben reputarse como un azote con que el omnipotente quiere afligirlos... considerando: que la propagación del fluido vacuno es de la mayor importancia y necesidad para evitar el funesto exterminio que causa la viruela...... acordó: nombrar un comisionado que recorra las provincias, deteniéndose a cada pueblo, para hacer dos o tres jornadas de vacunaciones; al partir de cada pueblo llevará en brazo la vacuna al pueblo inmediato, llevará un registro sobre número de vacunados, sexo, edad y luego darán cuenta cada dos meses a los subprefectos de la provincia..." ${ }^{21}$. Para los gobernantes, el concepto era que las epidemias son castigo de Dios, el control de las poblaciones a cargo de los autoridades de gobierno los prefectos, el control social a través de los cuerpos policiales, era posible en un escenario de guerras civiles; y las vacunas fueron ejercidas como elementos de control social en las poblaciones. Durante estos años, las noticias sobre epidemias que se registraban en Lima eran parte de lo cotidiano y mientras las instituciones médicas y sus miembros estaban en otras preocupaciones, habían dejado de ser un grupo tomado en cuenta para opinar. Se ha producido, al final de la década de 1820, la ruptura del grupo médico con la clase gobernante, un poder médico que se comenzó a construir en los años del virrey Abascal. Los iniciadores de la escuela de medicina $\mathrm{H}$ Unanue y

15 El curato era la célula básica de la administración civil y eclesiástica, por lo que los clérigos fueron personajes que acumularon poder político, religioso y económico. Eran funcionarios al servicio del Estado.

16 Oviedo Juan: Colección de Leyes, reglamentos y ordenes, del año 1821 a1860. Tomo VIII, pp. 321 Lima. Perú.

17 Oviedo J. Ob. cit. tomo VIII, p.322.

18 Oviedo J. Ob, cit. tomo VIII pp. 322-23.

19 Oviedo J. : ob. Cit. tomo VIII pp.327-28.

20 Defoe Daniel: Diario del año de la Peste. Colombia: Editorial Oveja Negra, 1985. 
Gregorio Paredes, entre otros, estaban más en labores políticas de estado $\mathrm{y}$, vinculado a los problemas de la organización de la república, sus seguidores no estuvieron a su altura y carecieron de propuestas que les permitiera como grupo profesional mantener una preponderancia.

\section{El Colegio de la Independencia y la primera protesta estudiantil}

El CI como institución del aparato gobernativo estaba sujeta a las disposiciones del prefecto departamental J. M. Egúsquiza, el cual con oficio le indica que debería asistir junto con todos los miembros de su corporación a la función religiosa que se hará en la catedral ${ }^{22}$. Estas disposiciones de asistencia a misa, ceremonias en palacio de gobierno, juramentaciones de nuevos gobiernos eran solicitadas por el prefecto al colegio con carácter obligatorio. Se repetían a lo largo de toda esta década, de acuerdo a los oficios encontrado en los archivos de la Facultad de Medicina. El prefecto de Lima, durante el año 1827, en quince oportunidades solicitó al CI que todos los profesores y alumnos acudan a ceremonias oficiales con la finalidad de "acompañar", "participar del júbilo" o "rendir homenaje"; y estas concurrencias eran obligatorias ${ }^{23}$.

La ausencia de un liderazgo y de capacidades organizativas llevaba a que la prefectura con circulares direccionara de acuerdo a sus intereses al CI. En diversos circulares le reclamaba “... Aún no he recibido contestación a la nota que dirijo a Ud. invitándole a un donativo voluntario ... de ese Colegio para cooperar en parte al armamento...." ${ }^{24}$. El CI era caja de resonancia de la prefectura para conducirlo a ceremonias, solicitarle donaciones voluntarias y decidir la vida académica, como lo demuestra el oficio del 23 julio 1829 dirigido al rector, donde le pide explicaciones "...qué motivos ha tenido para borrar a las matrículas de alumnos de su colegio a D Millas, y mientras tanto lo verifica, no se le considera excluido de la casa..." ${ }^{25}$. En el mismo archivo existen oficios del indicado prefecto, con fechas 27 y 28 de julio 1829 , sobre el mismo caso, en el cual el prefecto amenaza al rector y previene “...que se resuelva lo conveniente..." sobre el caso ${ }^{26}$. El prefecto era el representante del gobernante de turno y entre sus atribuciones estaba el mantener el orden social y formar columnas de adeptos, por lo que sus documentos expresaban las opiniones del gobernante sobre el papel del CI en la sociedad peruana. El CI era preso de los vaivenes de la inestable política de este periodo.

Al aparecer las salidas de estudiantes, a mediodía, para tomar alimentos en sus domicilios, tenían como consecuencia retraso en los estudios. Mediante oficio, el prefecto de Lima condena este hecho censurable y culpa a los rectores de este problema.

\section{Primera protesta estudiantil en medicina}

Las tendencias de una crisis continua del CI se dibuja desde 1821 y se cristalizarán a lo largo de esta década. A manera de anticipo exculpatorio, el gobierno, a través de la prefectura de Lima, remitió un oficio al rector llamándole la atención "...en lo sucesivo se abstenga de conceder a los alumnos de este colegio bajo ningún pretexto más días de asueto" ${ }^{27}$. Como una salida a la crisis y pretendiendo responder a los requerimientos del prefecto, la junta de profesores, en 1828, propuso al ministro eliminar algunas cátedras del área de ciencias básicas. Para protestar por tal decisión,

\footnotetext{
Diario El Conciliador, tomo III n ${ }^{\circ} 56$ del 18 de Julio 1832. AFM sin clasificar 25 diciembre 1826.

AFM no clasificados oficios de las fechas: 6 de febrero, 27 de marzo, 12 de abril, 26 y 31 de mayo, 3 y 13 de junio, 24 de julio, $5,7,11,16$, y 20 de agosto, 19 de septiembre y 25 diciembre de 1827 .

24 AFM no clasificados circulares de la prefectura del 26 y 31 de mayo de 1827.

25 AFM no clasificados oficio 23 julio 1829.

26 AFM no clasificados oficios del 27 y 28 de julio 1829.
} 
apareció en noviembre de 1828, en el Mercurio Peruano, una carta fechada en Cañete el 1 de noviembre, sin firma, pero que es posible hubiera sido escrita por $\mathrm{H}$. Unanue ${ }^{28}$. "Esta es una protesta pública contra la medida propuesta para que en el CI se enseñe sólo Medicina,.....aboliendo las cátedras de Matemáticas, Historia Natural, Farmacia, Química y de Física, como superfluas...”. Frente a esto, H Unanue argumentó “....es imposible emprender la Fisiología......sin saber la Física, ni ésta sin haber iniciado el estudio de las Matemáticas..." “...el estudio de Física lejos de ser superfluo es útil para la Medicina ..."; y termina la carta invocando a los forjadores del Colegio que pensaron la necesidad de formar médicos con una base científica. La carta anónima es una de las formas características de protesta social en colectividades con cierto desarrollo cultural ${ }^{29}$.

A esta situación de permanente crisis no fueron ajenos los estudiantes y éstos tomaron acciones, como lo demuestra el siguiente documento, cuya trascripción es de importancia por ser inédito ${ }^{30}$. Él nos revela las graves desavenencias en la administración y la carencia de autoridad, lo cual contribuyó a generar el deterioro del CI.

Los alumnos internos del CI acompañan una exposición de motivos sobre la conducta del rector.

"Los que suscribimos alumnos internos del colegio, que habiendo elevado al supremo conocimiento de la lamentable situación en que se encuentra el establecimiento a que pertenecen y de cuya condición no podrá salir mientras no se le encargue el rectorado a un sujeto de actitudes y conocimientos, porque el que actualmente lo dirige poco capaz en su desempeño, acompañan una breve exposición de los hechos que han marcado la conducta de este durante el tiempo que lo ejerce y en que apoyan su exposición...”

“...cuando en el año de 1826 el gran mariscal Santa Cruz separó al d.d. Mariano Fernandini del rectorado que ejerció por el abandono en que se hallaba el establecimiento en aquella época, fue encargado de él interinamente Nicolás Fernández de Piérola para que pusiese orden en los estudios y refaccionase el edificio que estaba bastante destruido; este recomendable ciudadano no cumplió en poco tiempo su encargo. En noviembre del mismo año fue nombrado José María Dávila para desempeñar el rectorado, pero en poco tiempo después dejó sentir el equivocado concepto que el gobierno tenía”.

“Desde el nombramiento del Dr. Dávila hasta la presente han señalado su conducta

1 ...su corta asistencia al colegio (sic), diariamente se cuenta a las 7 de la mañana, vuelve al medio día por algunos momentos, hora en que es inútil su presencia y se recoge después de las 10 de la noche...

2 ...su poco celo e interés por la instrucción( sic), ha consentido que se hayan presentado muchos exámenes sabiendo que las materias han sido mal dictadas...

3 ... descuido en la moral de los alumnos (sic), jamás corrige las faltas que se cometen aunque sean las de mayor gravedad.

4 ...la poca observancia del reglamento orgánico.

5 ...su falta de carácter, dignidad y respetabilidad... sufre las chansas [gestos] de algunos colegiales que se burlan de él...

7 ...la inmundicia, de la casa, en los lugares, en el traspatio de ella se notan muchas inmundicias, los cuartos se hallan destruidos atestados de basura.

Y que no habiendo hecho nada a favor del colegio en dos años y medio, .nada debe prometernos de él en adelante..........

...el único interés que nos ha obligado a pedir al jefe supremo de la republica la colocación de otro sujeto en el rectorado ha sido el sentimiento que tiene nuestro corazón al venir apreciando el abandono en que se halla el establecimiento......

27 AFM no clasificados, oficio del 28 de agosto 1827.

28 El Mercurio Peruano n ${ }^{\circ}$ 377. 1828 En dicho diario era aceptado la publicación de cartas en la sección variedades sin firma o con seudónimos.

29 Thompson Edward en Tradición y revuelta de clases . El delito del anonimato. Ha realizado un estudio detallado de diversas cartas publicadas entre 1750-1820; afirma que el anonimato era un mecanismo de refugio de los pobres y también de los profesionales.

30 AGN L. . 31, numero 23 ff. 10. 
firman. por la clase de patología : Manuel Aragón

por la cátedra de fisiología : José Bravo por la clase de anatomía: Pedro Espinoza, por la cátedra de filosofía: Juan A. Ribeyro por la cátedra de matemáticas: J. Roldán. Lima 8 de julio de 1829" 31 .

Esta protesta fue elevada al ministro del ramo, el cual requirió informe a los miembros de la junta de profesores. Éstos se reunieron el 31 de julio en la casa del Protomédico M Tafur. Estuvieron en la reunión José Maria Dávila, José Vergara, José Manuel Valdez, Manuel Seguín y Miguel de los Ríos, quienes acordaron, por unanimidad, se informe al gobierno, haciendo ver que dicho recurso sólo es producto de malignidad y de resentimientos particulares de los que firman. En el manuscrito aparecen las firmas de Miguel Tafur, José Manuel Valdez, Manuel Seguín, Miguel de los Ríos y José Vergara $^{32}$. El primero de agosto de 1829, el Ministro Álvarez puso el proveído "resérvese este expediente para cuanto se arregle el colegio".

El primer reclamo estudiantil en la historia de la Medicina en Perú fue llevado a cabo por los estudiantes de San Fernando como una preocupación por mejorar la crítica situación por la que atravesaba el CI. El estamento de los profesores optó por una defensa corporativa e ignorar el estado de cosas y no levantar alguna de las observaciones de los alumnos. Esta protesta es consecuencia de la acumulación de hechos vividos por los estudiantes y un silencioso proceso que sale a la luz con todos los riesgos y consecuencias que podrían haberse derivado. No se conoce si tuvieron algunas consecuencias en los dictados de cursos y/o represalias sobre los alumnos, pero lo cierto es que continuó en su cargo el referido rector.

Este manuscrito, desconocido hasta la fecha, es una crítica severa a la metodología de enseñanza; pone de manifiesto que no sólo eran problemas económicos y habla más de problemas educativos y administrativos. De acuerdo a esto, es cierto la existencia de apremios económicos, pero el grupo directivo estaba en otras preocupaciones antes que en seguir el derrotero marcado por $\mathrm{H}$ Unanue. La lectura del pliego de reclamaciones habla de lecciones teóricas, librescas y memorísticas y de un desorden administrativo y ausencia de una higiene mínima para un colegio de medicina. La respuesta de los catedráticos lleva a sostener lo que en líneas anteriores afirmamos, que el grupo de profesores carecía de un norte y era un grupo que rompió la continuidad necesaria para implementar las propuestas que el plan de estudios inicial había impulsado.

Las propuestas de las autoridades educativas estaban en un plano diferente al de los reclamos de $\mathrm{H}$ Unanue. Las protestas de los estudiantes y de su fundador no fueron suficientes para cambiar el rumbo de un colegio que al fin de esta década había tocado fondo y será un remedo de males educativos de la enseñanza virreinal. Habían cambiado los hombres y las épocas; pero, las actitudes y los hechos eran de corte tradicional, la independencia y la república habían sido nada más que literatura, sin continuidad en las ideas del plan original propuesto por $\mathrm{H}$ Unanue. La protesta estudiantil no tuvo repercusiones en los medios escritos en la sociedad límense. Hemos revisado los diarios de la época, publicados en Lima sin encontrar nota alguna al respecto ${ }^{33}$.

\section{Conclusiones}

1. Los años iniciales de 1821 a 1830 fueron de una marcada crisis económica y educativa en el CI. Las continuas crisis fiscales determinaron que el plan de estudios del Colegio Real de Medicina y Cirugía de Lima fuera dejado de

31 En el año académico de la protesta, éstos eran todos los cursos que se dictaba; entre los delegados se encontraba José Riveyro, alumno becario desde 1826 .

32 Nótese que no participa de la reunión Cayetano Heredia, quien en el momento era disector de Anatomía; podría interpretarse de una jerarquía entre los profesores. 
lado suprimiéndose los cursos de humanidades.

2. Las continuas crisis de gobierno y guerras civiles de los inicios de la república repercutió en los alumnos y profesores. La continuidad del grupo académico fue rota y el grupo continuador careció de liderazgo y propuestas creativas.

3. Al final de la década, en 1829 , se va a realizar la primera protesta estudiantil de estudiantes de medicina, aduciendo problemas académicos, administrativos, reclamos que no fueron escuchados por una reacción corporativa de los profesores.

4. El prefecto departamental de Lima ejerció influencia política, académica y sobre la marcha administrativa del Colegio de la Independencia.

\section{Bibliografía}

- Aljovín C. Caudillos y Constituciones en el Perú 1821-1845. Ed Fondo de Cultura Económica PUCP. Lima, Perú 2000;p 40.

- Paz Soldán CE. La escuela médica peruana. Ed del Hospital Víctor Larco Herrera. Lima, Perú 1911; p 32-3.

- Lastres J. Historia de la medicina peruana. La medicina en la República. Universidad Nacional Mayor de San Marcos Imprenta Santa María. Lima, Perú 1951; p 77.

- Valdizán H. La facultad de medicina de Lima 1811-1911. Imprenta Sanmarti y Cia. Lima, Perú 1913; p 147.

- Basadre J.Historia de la República. Tomo I, 7 ma edición. Editorial Universitaria. Lima, Perú 1993; 136.

- Defoe D. Diario del año de la peste. Editorial Oveja Negra. Colombia 1985.

- Thompson E. En: Tradición y revuelta de clases. El delito del anonimato. Editorial Crítica. Barcelona 1995.

- Oviedo J.Colección de leyes, decretos y ordenes. Tomo VIII. Lima Perú 1886.

- Archivo General de la Nación. Colegio de la Independencia. Lima, Perú 10 de enero de 1827. Legajo 11 cuaderno 29 ff. 3.

- Archivo General de la Nación. Colegio de la Independencia. Lima, Perú 8 de enero de 1827. Legajo 11 ff. 2.

- Archivo General de la Nación. Colegio de la Independencia. Lima, Perú 13 de noviembre 1828. Legajo 24 cuaderno 119 ff. 1.

- Archivo General de la Nación. Colegio de la Independista. Lima, Perú 20 y 23 de junio de 1829. Legajo 30 cuaderno 94 ff. 3.

- Archivo General de la Nación. Colegio de la Independencia. Lima, Perú 6 de diciembre de 1828. Legajo 25 número 54 ff. 3.

33 Diarios revisados: El Mercurio Peruano, El Rímac, El Liberal, El Republicano.
- Archivo General de la Nación. Legajo 30 número 9 ff 2 del 23 julio 1829 y Legajo 34 número 245 ff 2 del 24 de abril Lima, Perú 1829.

- Diario El Conciliador. Tomo III nº 56. Lima, Perú 18 de julio 1832.

- Archivo Facultad de medicina [no clasificados]. Circulares de la prefectura. Lima, Perú 26 y 31 de mayo de 1827.

- Archivo Facultad de medicina [noclasificados]. Lima, Perú. Oficio 23 julio 1829.

- Archivo Facultad de medicina [no clasificados]. Lima, Perú .Oficios del 27 y 28 de julio 1829.

- Archivo Facultad de medicina [no clasificados]. Lima, Perú. Oficio del 28 de agosto 1827.

- El Mercurio Peruano. nº 377. Lima, Perú 1828. 